\title{
La crisis económica y sus efectos en las instituciones de educación superior: el caso de la UNAM
}

Eduardo Salas Estrada ${ }^{1}$

\section{RESUMEN}

Este ar tí cu lo está ba sa do en la re co men da ción que hace la UNESCO, para que los Es ta dos m iem bros de esta or ga ni za ción des ti nen el $8 \%$ del Producto Interno Bruto (PIB) a la educación.

Se ana li za la dis tri bu ción delPIB en tre los tres sec to res bá si cos (agri cul tu ra, in dus tria y ser vi cios) en Mé xi co y se com pa ran los gas tos en educación de nuestro país con otras naciones de América Latina en relación con el PIB.

Fi nal men te se ana li za el pre su pues to de la UNAM ejer ci do en los años 1981, 1982, 1988, 1989 y se compara con un documento de la Co or di na ción Na cio nal par la Pla nea ción de la Edu ca ción Su pe rior (CONPES) para el pe rio do 1981 - 1991. Se des ta ca el no ta ble apoyo que ha recibido la investigación bibliotecológica en la UNAM, en el mismo periodo.

\section{ABSTRACT}

The article is based on the recommendation made by the UNESCO that its member states assign $8 \%$ of their NBP to education. An analy sis is made of the NBP among the three ba sic sec tors (agri cul tu re, in dus try and ser vices) in Me xi co and the budger for edu ca tion in Mexico is compared to other Latin American countries in relation to the NBP.

Lastly, an analysis is made of the budget for the UNAM for the years 1981, 1982, 1988 and 1989 and the re sults com pa red to the do cument issued by the Coordinación Nacional para la Planeación de la Educación Superior (CONPES) for the period 1981-1991. The support for research in librarianship in UNAM has receibed a remarkable increase in the same period.

En los úl ti mos años se ha he cho más fre cuen te que di ver sas personalidades de la in ves ti ga ción cien tífica se pronun cien en contradela políticagubernamental de recordarsis te máticamentelos pre su pues tos de las ins ti tu cio nes de edu ca ción su pe rior en nuestro país, y el de nuestra casa de estudios en particular.

En tre los más des ta ca dos in ves ti ga do res de la UNAM se en cuentran René Drucker, ${ }^{2}$ To más Bro dy, Ruy Pé rez Ta ma yo y Jor ge Flores Val déz. ${ }^{5}$ En ge ne ral to dos la men tan la fal ta de apo yo a la investigación científica y tec noló gi ca, y hacenalu sión repe tidamente a la falta de observancia por parte de las autoridades responsables de la planeación nacional, a la recomendación de la UNESCO en el sentido de destinar el $8 \%$ del Producto Interno Bruto a la educación.

El pro duc to In ter no Bru to (PIB) y su dis tri bu ción en tre los sec tores agrí co la, in dus trial y de ser vi cios a pre cios de 1980, en mi llones M.N. ha sido el que se muestra a continuación:

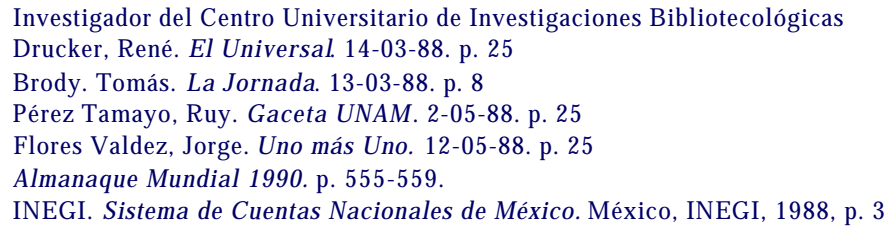

\begin{tabular}{|l|c|c|c|c|}
\hline & 1982 & 1984 & 1986 & 1987 \\
\hline PIB (Mills) & 4,591 & 4,500 & 4,725 & 4,793 \\
\hline${ }^{* *}$ Agricultura & 8 & 7 & 8.5 & 8.5 \\
\hline${ }^{* *}$ Industria & 37 & 38 & 27.1 & 27.3 \\
\hline${ }^{* *}$ Servicios & 55 & 55 & 64.4 & 64.2 \\
\hline
\end{tabular}

** Dis tribución Porcentual

Fuentes: Almanaque Mundial 1990 p. $179^{6}$; INEGI. Sistema de Cuentas Nacionales de México (Estimación preliminar; 1987 , p. 3$)^{7}$ 
La dis tri bu ción del pro duc to in ter no bru to en tre los tres sec to res prin ci pa les, mues tra que el sec tor de la agri cul tu ra se ha man te nido constante, el sector industrial ha sufrido una reducción, en tanto que los servicios, dentro del cual se ubica la educación ha recibido un incremento en los últimos años.

El di rec tor delCONACYT en su in for me de $1987,{ }^{8}$ afir ma ba que el gas to to tal en cien cia y tec no lo gía, os ci la ba al re de dor del $0.5 \%$ del ProductoInternoBruto.(Cuandose es perabaquellegaraal $1.5 \%$ )

Según información del Almanaque Mundial 1990. ${ }^{9}$ Los gastos de edu ca ción por par te del Go bier no Cen tral de los dis tin tos países de América Latina han sido los siguientes, tomando como base el Producto Interno Bruto:

\begin{tabular}{|l|c|c|}
\hline \multicolumn{1}{|c|}{ Pais } & Ano & Porcentaje \\
\hline Mexico & 1986 & \\
\hline Costa Rica & 1986 & 25.7 \\
\hline El Salvador & 1984 & 10.8 \\
\hline Guaternala & 1987 & 23.1 \\
\hline Honduras & 1985 & 24.0 \\
\hline Nicaragua & 1986 & 15.5 \\
\hline Panama & 1986 & 15.4 \\
\hline Argentina & 1986 & 7.5 \\
\hline Bolivia & 1986 & 18.8 \\
\hline Brasil & 1986 & \\
\hline Colombia & 1986 & 36.2 \\
\hline Chile & 1986 & 17.3 \\
\hline Ecuador & 1983 & 25.8 \\
\hline Paraguay & 1986 & 18.8 \\
\hline Perú & 1985 & 17.9 \\
\hline Uruguay & 1986 & 14.9 \\
\hline Venezuela & 1985 & 29.3 \\
\hline
\end{tabular}

Por su parte el Se cre ta rio de Edu ca ción Pública ${ }^{10}$ de cla ró que en 1982 el sectoreducativo con tó con un pre su pues to de 2,256 millo nes de pe sos y que en 1988 éste ha bía as cen di do a 460,000 millo nes de pe sos, re pre sen tan do un au men to del $4.5 \%$ en el po der adquisitivo real a precios de 1982 .

Para tra tar de en ten der este pro ble ma, to ma re mos como base un trabajo de la Coordinación Nacional para la Planeación de la Educación Superior (CONPES) en el que se contemplaban dos al ter na ti vas de pla nea ción pre su pues tal para la dé ca da de 1981 a 1991 , ${ }^{11}$ te nien do como base los por cen ta jes que en 1981 se de dicaban a diversos aspectos de la educación:

Los ejercicios presupuestales de los años 1981,1982,1988 y 1989 en la UNAM fueron los siguientes:

\begin{tabular}{|l|c|c|c|}
\hline & 1981 & $\begin{array}{c}\text { Alter- } \\
\text { nativa A }\end{array}$ & $\begin{array}{c}\text { Alter- } \\
\text { nativa 8 }\end{array}$ \\
\hline Docencia & $68 \%$ & $44 \%$ & $58 \%$ \\
\hline Administración & $20 \%$ & $15 \%$ & $12 \%$ \\
\hline Investigación & $4 \%$ & $20 \%$ & $15 \%$ \\
\hline Difusión Cultura & $5 \%$ & $12 \%$ & $8 \%$ \\
\hline Bibliotecas & $1 \%$ & $5 \%$ & $4 \%$ \\
\hline $\begin{array}{l}\text { Formación y } \\
\text { Actualización de }\end{array}$ & $2 \%$ & $4 \%$ & $3 \%$ \\
Profesores & & & \\
\hline
\end{tabular}

** Es tos ru bros se in clu yen en otras par ti das, por lo que no es po si ble desglosarlos

\begin{tabular}{|c|c|c|c|c|}
\hline & $1981^{4 t}$ & $1982^{(2)}$ & $1988^{+2 \pi}$ & $1989^{16}$ \\
\hline Docencia & $62.7 \%$ & $62.5 \%$ & $64.6 \%$ & 64.765 \\
\hline Administración & $11.1 \%$ & $10.99 \%$ & $5.5 \%$ & $5.38 \%$ \\
\hline Investigación & $17.2 \%$ & $17,3 \%$ & $21.3 \%$ & $21.88 \%$ \\
\hline Dfusion Cultural & 8.74 & $9.0 \%$ & $7.9 \%$ & 8.00\% \\
\hline \multicolumn{5}{|l|}{ "Bbliotecas } \\
\hline $\begin{array}{l}\text { "Formacion y } \\
\text { Actualuzacion de } \\
\text { Profesores }\end{array}$ & & & & \\
\hline
\end{tabular}

En los años 1981 y 1982 el resumen de egresos de la UNAM mues tra una ten den cia más cer ca na a la al ter na ti va "B" plan teada por el CONPES. Se nota que en los ru bros de do cen tes e in vestigación se han dado incrementos de $2.1 \%$ en el primero y de $4.5 \%$ en el se gun do, sin em bar go a la luz de los da tos, de 1989 se observa que los rubros docencia e investigación prácticamente se han estabilizado.

Un as pec to que vale la pena des ta cas es el he cho de que mien tras los incrementos en las partidas presupuestales globales destinadas a la do cen cia y la in ves ti ga ción son mo de ra dos, la in ves ti gación bi blio te co ló gi ca en la UNAM ha re ci bi do un no to rio apo yo presupuestal, que le permitirá crear una sólida infraestructura para sus actividades según puede observarse en el cuadro siguien te, En él se mues tran las ci fras por cen tua les asig na das, tomando como base el presupuesto para el año 1985.

Es im por tan te des ta car que el pro me dio de los in cre men tos presupuestales, con base en el presupuesto asig na do para el año inmediato anterior, es de $208.5 \%^{16}$

Esto nos lleva a con si de rar que los es fuer zos de pla nea ción econó mi ca de la Na ción han mar ca do las di rec tri ces para el de sa rrollo de la educación nacional en general, del cual la educación superior y en particular la UNAM, no puede estar ajena.

8 "Informe del CONACYT" Proceso. 8-02-88. . p. 18-20.

9 Almanaque Mundial 1990. p. 555-559.

10 González Avelar, Miguel. Proceso. 8-02-88. p. 18-20

11 CONPES. El desarrollo de la Educación Superior 1981-1991. México, ANUIES/SEP,1982. P. 24-25.

12 Anuario Estadístico. UNAM, 1981. p. 229

13 Información proporcionada amablemente por la Secretaría Administrativa del CUIB.

14 Programa para la modernización educativa 1989-1994. México, Secretaría de Educación Pública. 1989.

15 Martínez C., Néstor. "El presupuesto de la UNAM casi 1.2 Billones; deterioro paulatino" Uno más Uno 14-02-90, p.1-7.

16 Información proporcionada amablemente por la Secretaría Administrativa del CUIB. 


\begin{tabular}{|c|c|}
\hline Año & $\begin{array}{c}\text { \% Incrementado respecto al } \\
\text { año anterior }\end{array}$ \\
\hline 1985 & 100.0 \\
\hline 1986 & 192.8 \\
\hline 1987 & 236.7 \\
\hline 1988 & 288.4 \\
\hline 1989 & 116.1 \\
\hline
\end{tabular}

Por otra parte, un indicador básico para la planeación educativa en el mar co de la pla nea ción na cio nal de cual quier país, es aquél establecido por la UNESCO que recomienda que del Producto In ter no Bru to se des ti ne el $8 \%$ a la edu ca ción, cri te rio que en Méxi co no se cum ple. Más aun, la caí da del Pro duc to In ter no Bru to se debe en tre otras cau sas a la fal ta de apo yo a la in ves ti ga ción y a la edu ca ción en di fe ren tes ni ve les y en am bien tes di ver sos en lo económico y en lo social, que requieren ser organizados en un sis te main te gra do no sólo a los pla nes de de sa rrollo eco nó mi co y so cial del país, sino como un pro yec to in te gral de de sa rro llo cultural de nuestro país.

La falta de apoyo a la investigación, es un hecho real que está propiciando que un alto porcentaje de becarios no regresen al país al con cluir sus es tu dios en el ex tran je ro y que mu chos in vestigadores abandonen la Uni versi dad para irse a pres tar ser vi cios a la iniciativa privada tanto nacional como transnacional.

Este he cho pro vo ca que la po lé mi ca en tre in ves ti ga do res y au toridades universitarias se refiera casi siempre a la investigación básica, aplicada y a la investigación tecnológica; haciendo poca o nula re fe ren cia res pec to a la ne ce si dad de crear un pro yec to armóni co que in te gre lain ves ti ga ción cien tífica con la hu manís tica y la artística.

Es de suma im por tan cia con ser var y acre cen tar el ca rác ter mul tidis ci pli na rio del trabajocien tífico en nues tracasa de es tu dios, el cual re quie re de im por tan tes apo yos para la in te gra ción ver da derade lacomunidadcien tífica, materializadosés tos en equi pos de laboratorio adecuados, personal auxiliar, y publicaciones especializadas que propicien la difusión de los avances de las ciencias bá si cas, de la in ves ti ga ción tec no ló gi ca y su re la ción con las ac ti vi da des pro duc ti vas, así como de las cien cias so cia les, de las humanidades y las expresiones artísticas.

Sin embargo, es imperativo que para lograr todo esto, la educación en general y la educación superior en particular, reciban la aten ción pre pon de ran te que les co rres pon de en la pla nea ción nacio nal y que ésta se ma ni fies te en el otor ga mien to de ma yo res recursos económicos a esta actividad. Es conveniente reconocer que la posibilidad de alcanzar los mejores niveles está en relación directa con el incremento del Producto Interno Bruto de nuestro país, lo cual es un reto para nuestra sociedad actual, sin ex cep ción de al gún sec tor. La edu ca ción es par te in te gral del planea mien to eco nó mi co y so cial, por tan to la pro mo ción de la edu- ca ción y de la in ves ti ga ción a dis tin tos ni ve les y en am bien tes diversos requiere de una planeación y organización integrada al desarrollo económico y social de toda nación.

La pla nea ción de la edu ca ción su pe rior du ran te los úl ti mos cin co años se ha realizado con una tendencia moderada de cambios, afectada prin ci pal men te por la re la ción peso/dó lar. El ejer ció rigu ro so del pre su pues to en nues tra casa de es tu dios, mues tra también ajustes en sus diversos rubros debido a los recortes que ha su fri do el sub si dio del Go bier no; sin em bar go es cla ro que el rubro de la investigación es el que más se ha intentado fortalecer, como queda de manifiesto con el incremento de cuatro puntos por cen tua les que han ga na do en tre 1982 y 1988 . Este avan ce sin embargo sees ti main su fi cien te si se com para con el pre su pues to de 1989 donde se observa que los porcentajes se estabilizaron, in di can do una pe li gro sa ten den cia alsta tu quo que es a to das luces injusto, principalmente para el sector de investigación. Por esta razón se ha vuel to un cla mor in con te ni ble en tre la co mu nidad cien tífi ca el que se in cre men te el pre su pues to a la in ves ti gación. Esperamos que como resultado de las deliberaciones y acuerdos emanados del Congreso Universitario efectuado en mayo de 1990, el Go bier no ca na li ce los re cur sos ne ce sa rios para el apoyo de los programas que reactiven la investigación en la UNAM.

Por úl ti mo, se gún el Pro gra ma Na cio nal de De sa rrol lo de la Edu cación Superior $^{176}$ señala que en 1970 y 1989 las cifras de la Educación superior eran las siguientes:

\begin{tabular}{|l|c|c|c|}
\hline & 1970 & 1989 & $\begin{array}{c}\text { Porcentaje de } \\
\text { incremento }\end{array}$ \\
\hline Alumnos & 271,275 & $1,256,942$ & 463 \\
\hline Maestros & 25,056 & 128,481 & 512 \\
\hline $\begin{array}{l}\text { Establecimien- } \\
\text { tos }\end{array}$ & 385 & 1,999 & 519 \\
\hline
\end{tabular}

En la UNAM la situación para años similares es la siguiente:

\begin{tabular}{|l|c|c|c|}
\hline & 1973 & 1989 & $\begin{array}{c}\text { Porcentaje de } \\
\text { incremento }\end{array}$ \\
\hline Alumnos & 198,479 & 269,694 & 135.9 \\
\hline Maestros & 14,697 & 29,079 & 197.8 \\
\hline Presupuesto & $960^{\circ}$ & $997,631^{\circ}$ & $1,039.1$ \\
\hline
\end{tabular}

*Millones de pesos

Otra fuente ${ }^{187}$ señala que a programas de investigación sólo se des ti na el $0.4 \%$ del PIB, que el $90 \%$ de es tos re cur sos pro vie nen de la Federación y que la mayor parte se utiliza para el pago de salarios, pero a pesar de esto los honorarios tanto de profesores como de in ves ti ga do res son a to das lu ces in su fi cien tes en el marco de las condiciones actuales de la economía nacional. 


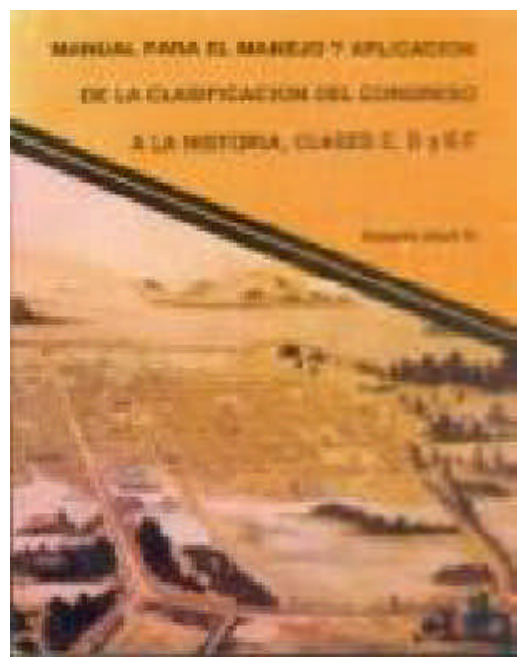

Ma nual para el ma ne jo y la aplicación de la Clasificación del Congreso a la Historia clases $C, D$ y $E, F$. 